\title{
Visualization of exosome-mediated miR-210 transfer from hypoxic tumor cells
}

\author{
Kyung Oh Jung ${ }^{1,2,3,5}$, Hyewon Youn ${ }^{1,3,4}$, Chul-Hee Lee ${ }^{1,2,3}$, Keon Wook Kang ${ }^{1,3}$, June- \\ Key Chung ${ }^{1,2,3}$ \\ ${ }^{1}$ Department of Nuclear Medicine, Seoul National University College of Medicine, Seoul, Korea, 110-799 \\ ${ }^{2}$ Department of Biomedical Sciences, Seoul National University College of Medicine, Seoul, Korea, 110-799 \\ ${ }^{3}$ Cancer Research Institute, Seoul National University College of Medicine, Seoul, Korea, 110-799 \\ ${ }^{4}$ Cancer Imaging Center, Seoul National University Hospital, Seoul, Korea, 110-799 \\ ${ }^{5}$ Current affiliation: Department of Radiation Oncology \& Medical Physics, Stanford University, CA, USA, 94305 \\ Correspondence to: Hyewon Youn, email: hwyoun@snu.ac.kr \\ June-key Chung, email: jkchung@snu.ac.kr \\ Keywords: exosome, breast cancer, miR-210, tumor microenvironment, hypoxia \\ Received: August 01,2016 Accepted: December 01,2016 Published: December 27, 2016
}

\section{ABSTRACT}

Cancer cells actively release exosomes carrying specific cellular components, such as proteins, mRNA, and miRNA, to communicate with various cells in the tumor microenvironment. We visualized exosome-mediated transfer of miR-210 from hypoxic breast cancer cells to neighboring cells using a miR-210 specific reporter system. By in vitro and in vivo visualization, we found that exosomes with miR-210 were transferred to cells in the tumor microenvironment and that miR-210 was involved in expression of vascular remodeling related genes, such as Ephrin A3 and PTP1B, to promote angiogenesis. These results indicate that cellular components, such as miRNAs from hypoxic cancer cells, spread to adjacent cancer cells in the tumor microenvironment via exosomes and influence tumor progression.

\section{INTRODUCTION}

Hypoxia is one of the hallmarks of cancer $[1,2]$. Cells within the tumor become hypoxic as the tumor mass increases, resulting in activation of HIF-1 $\alpha$ to induce various malignant phenotypes. Hypoxic tumors become resistant to chemotherapy and radiotherapy; therefore, imaging hypoxia is important in cancer diagnostics and therapeutic planning [3, 4].

Recently, some microRNAs have been considered cancer biomarkers because of their important roles in regulating gene expression [5]. MicroRNAs are singlestranded, non-coding small RNAs that regulate degradation or post-translational inhibition of target mRNA by partially or completely binding to the $3^{\prime}$ untranslated region (UTR) of the mRNA [6]. Among the possible cancer biomarker candidates, miR-210 levels have been reported to be highly increased in hypoxic cells and may be involved in tumor growth and angiogenesis $[7,8]$.

As microvesicles (30-100 $\mathrm{nm}$ in diameter) secreted by cells, exosomes contain many functional proteins, mRNAs, and miRNAs, and play a role in intercellular communication $[9,10]$. Recently, the roles of exosomes in the tumor microenvironment have been emphasized for their involvement in tumor progression, angiogenesis, and metastasis [11-13]. Several studies have shown that miR-210 levels in hypoxic cells and the relative amount of secreted exosomes from hypoxic cancer cells are highly increased $[14,15]$. However, exosome-mediated transfer of miR-210 to neighboring cells and the effects of hypoxic exosomes on the tumor microenvironment have not been clearly elucidated.

In this study, we constructed a reporter gene vector with triple seed sequences to visualize the presence of miR-210 and investigated the effect of exosome-mediated transfer of miR-210 in the tumor microenvironment using reporter expressing cells.

\section{RESULTS}

\section{Characterization of exosomes and in vivo} biodistribution

To characterize exosomes, ultra-centrifugation and the ExoQuick kit were used for purification. Transmission Electron Microscopy and NanoSight revealed the presence 
of microvesicles from both isolation methods, and their morphology and size were within the expected range of an exosome ( $100 \mathrm{~nm})$ (Figure 1a). Purified exosomes had exosome markers, including CD9, CD63, and Alix. The cellular marker calnexin was not observed (Figure 1b).

To visualize the biodistribution of exosomes in mice in vivo, we isolated exosomes from 4T1 cells, labeled them with $\mathrm{Cy} 7$ fluorescent dye, and collected images after intravenous injection into 4T1 tumor models. Cy7exosomes (Supplementary Figure 1) accumulated in 4T1 tumors in vivo and ex vivo (Figure 1c). To investigate systemic transfer of exosomal miR-210 in the blood circulation, we directly injected DFO $(200 \mu \mathrm{M})$ into $4 \mathrm{~T} 1$ tumor grafts in mice and isolated exosomes from the serum. We observed a significant increase in the amount of miR-210 from exosomes isolated from the serum of DFO-

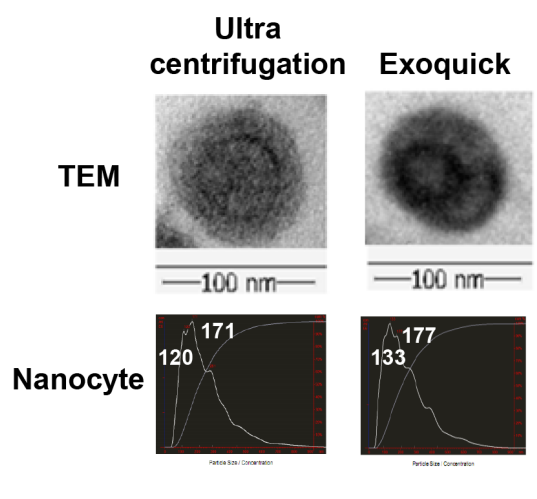

b

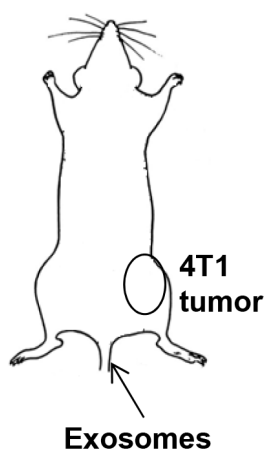

From 4T1 cells
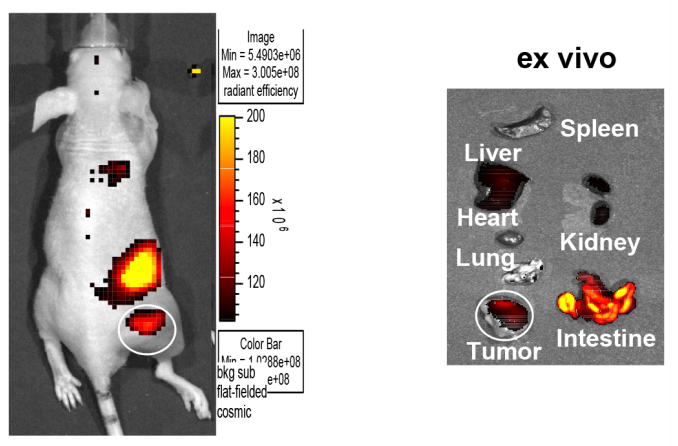

d

Exosomes from serum

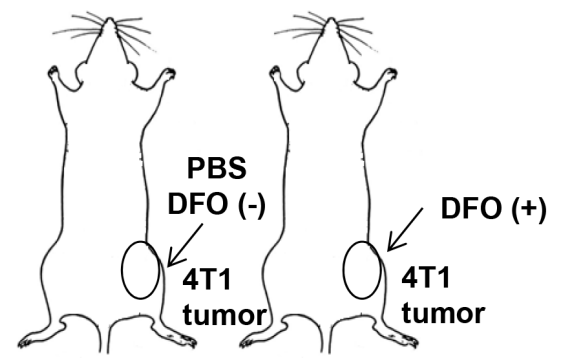

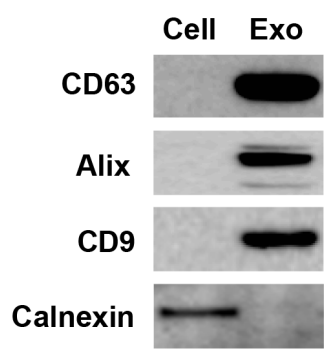

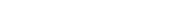


treated mice (3.71-fold; $\mathrm{P}=0.0368)$, indicating systemic circulation of exosomes containing miR-210 (Figure 1d).

Because we used DFO to induce hypoxia, we evaluated the cytotoxicity of DFO in 4T1 cells (Supplementary Figure $2 \mathrm{a}$ ). Less than $400 \mu \mathrm{M}$ of DFO was considered non-toxic to $4 \mathrm{~T} 1$ cells.

\section{DFO-induced hypoxia and exosomes in a hypoxic environment}

To compare DFO-mediated hypoxia and natural hypoxia, we measured HIF-1 $\alpha$ levels with and without DFO treatment. HIF-1 $\alpha$ protein levels were increased in DFO treated $(+)$ cells and exosomes compared to the control (Figure 2a). The amount of secreted exosomes in hypoxic cells was measured from the culture media of 4T1 cells with or without DFO treatment. Increased exosome secretion was observed in the medium of DFO
$(+)$ cells compared to DFO (-) cells (1.40-fold, $\mathrm{P}=$ 0.0047, Figure 2b).

Based on real-time quantitative PCR (Figure 2c), cellular HIF-1 $\alpha$ expression levels in DFO $(+)$ cells were increased after DFO treatment compared with DFO (-) cells (2.73-fold, $\mathrm{P}=0.00403)$. However, there was no significant difference in exosomal HIF-1 $\alpha$ levels regardless of DFO treatment. Cellular and exosomal miR210 levels in DFO $(+)$ cells showed a significant increase (Figure 2d) compared with DFO (-) cells (15.70-fold, P = 0.0184 for cellular miR-210; 12.73-fold, $\mathrm{P}=0.0023$ for exosomal miR-210).

\section{Imaging miR-210 activation in hypoxic cancer cells}

To visualize miR-210 in cells, we designed a luciferase-based miR-210 reporter vector that contains
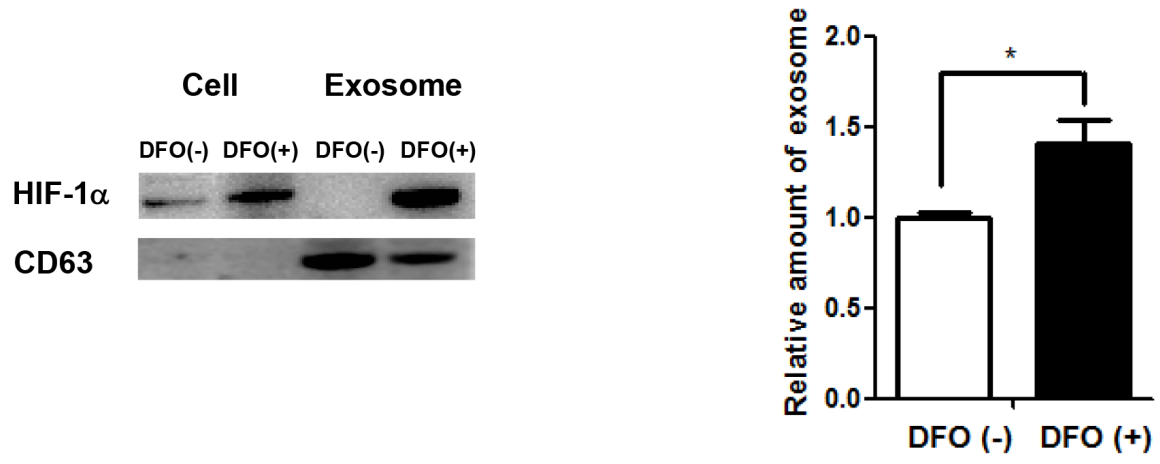

C

d
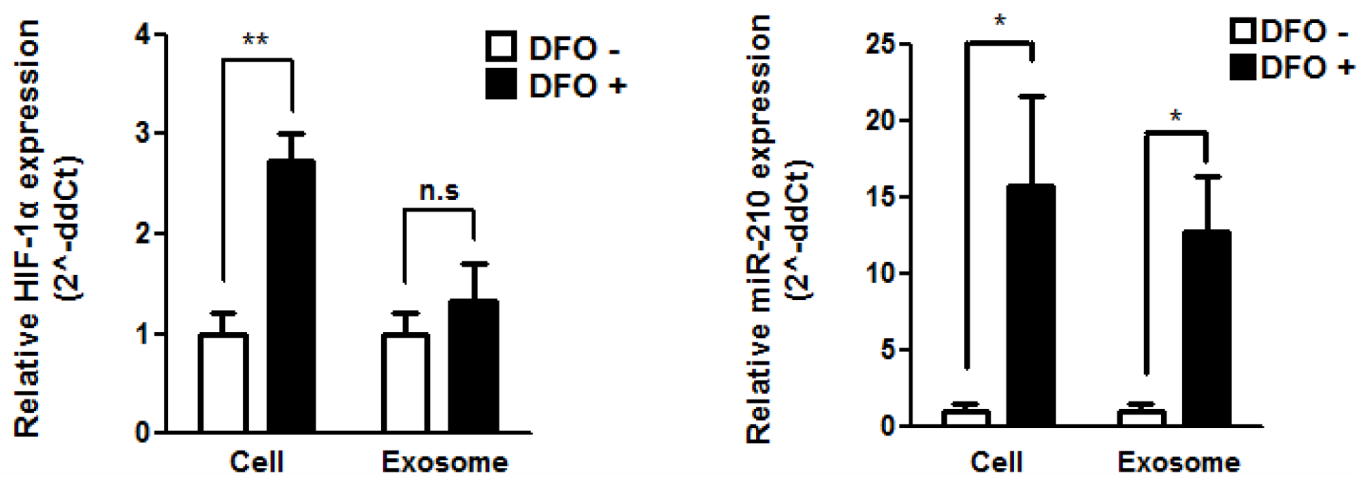

Figure 2: DFO-induced hypoxia and exosomes in a hypoxic environment. a. Induction of a hypoxic environment with DFO ( $400 \mu \mathrm{M}, 48 \mathrm{hr}$ treatment) and the amount of Hif-1 $\alpha$ as evaluated by western blot. b. Relative amount of exosomes produced by $4 \mathrm{~T} 1$ cells after treatment with DFO. c. HIF-1 $\alpha$ expression in cells and exosomes after treatment with DFO. d. miR-210 expression in cells and exosomes after DFO treatment. $* \mathrm{P}<0.05$. 
three repeated miR-210 target sequences (CGCACA) to amplify sensitivity to miR-210 binding (Figure 3a). In this reporter system, miR-210 binding can turn off the luciferase signal due to formation of double stranded RNA. To test the miR-210 reporter function of this vector, we established a 4T1 cell line expressing a miR-210 reporter (4T1/miR210). We induced miR-210 with DFO treatment in the reporter expressing cells and evaluated luciferase activity. Both signals from IVIS imaging and the luciferase activity determined from an enzymatic assay were decreased in a DFO dose-dependent manner (Figure 3b). In bioluminescent imaging of cells (Figure $3 \mathrm{c})$, signals from DFO $(+)$ cells were decreased compared to those from DFO (-) cells $(0.22$-fold, $\mathrm{P}=0$. 0057). For in vivo imaging, DFO was injected directly into the 4T1/ miR210 tumor in mice. Luciferase signals from the tumor were decreased after DFO treatment, whereas signals from tumors treated with PBS as a control were similar before and after treatment. Luciferase signals from DFO $(+)$ cells were decreased by 0.53 -fold $(\mathrm{P}=0.0271)$ compared to those from DFO (-) cells (Figure 3d). From IHC of tissues (Figure 3e), we observed that luciferase expression in DFO $(+)$ tumors was decreased and HIF-1 $\alpha$ expression in DFO $(+)$ tumors was increased compared to DFO (-) tumors.

\section{Imaging uptake of exosomes by cells in the tumor microenvironment}

To confirm uptake of exosomes in the tumor microenvironment, exosomes were labeled with DiI/ or $\mathrm{DiO}$ and imaged with the Maestro ${ }^{\mathrm{TM}}$ fluorescence imaging system and confocal microscopy (Supplementary Figure 3a). Fluorescence-labeled exosomes were exposed to various cells in the tumor microenvironment, such as tumor cells (4T1), endothelial cells (SVEC), macrophages (Raw264.7), stem cells (mBs-MSC), fibroblasts (3T3), and dendritic cells (JAWS2). In confocal microscopy, uptake of fluorescently stained exosomes in various cells was also observed (Supplementary Figure 3b). To image exosomes using another method, we constructed a CMV-driven GFP/ RFP-tagged CD9 vector using the well-known exosomal marker protein CD9. In both confocal microscopy and Maestro images, we were able to image the fluorescence expressing exosomes in the CD9-GFP/RFP vectortransfected 4T1 cells (Supplementary Figure 4a, 4b).

\section{Imaging of exosome-mediated transfer of miR- 210 to recipient cancer cells in vitro and in vivo}

To assess miR-210 transfer through exosomes, we isolated exosomes from DFO-treated 4T1 cells (hypoxic exosomes) and added them to $4 \mathrm{~T} 1 / \mathrm{miR}-210$ or SVEC/ miR-210 cells for in vitro evaluation. We also injected exosomes from DFO-treated/or non-treated 4T1 cells (designated as EXO (+)/or EXO (-)) to grafted tumors with $4 \mathrm{~T} 1 / \mathrm{miR}-210$ to visualize exosome-mediated transfer of miR-210 in vivo (Figure 4a). When we exposed hypoxic exosomes to 4T1/miR-210 and SVEC/miR-210 cells, IVIS images and luciferase activity assay showed that luciferase signals in both cells were significantly decreased in a dose-dependent manner (Figure 4b). Cytotoxicity of the exosome treatment was also evaluated in both 4T1 and SVEC cells (Supplementary Figure 2b, 2c). Even with an increased amount of exosomes, treatments were not cytotoxic to $4 \mathrm{~T} 1$ and SVEC cells.

After $48 \mathrm{~h}$ treatment with exosomes, luciferase signals from Exo $(+)$ 4T1 cells were decreased 0.67fold $(\mathrm{P}=0.0262)$ compared to Exo (-) 4T1 cells (Figure 4c). Luciferase signals from Exo $(+)$ SVEC cells were also significantly decreased 0.27 -fold $(\mathrm{P}=0$. 0005) compared to Exo (-) SVEC cells. To measure the effect of hypoxic exosomes on SVEC cells, wound healing assays were performed. We observed that migration of Exo (+) SVEC cells was higher than in Exo (-) SVEC cells (Supplementary Figure 5a), and capillary-like structures were increased in Exo (+) SVEC cells (Supplementary Figure 5b). Furthermore, proliferation of Exo (+) SVEC cells was increased under a prolonged incubation time (Supplementary Figure 5c).

In bioluminescence imaging of grafted tumor models (Figure 4d), luciferase signals in the Exo $(+)$ tumor were also decreased after exosome treatment, while signals in Exo (-) tumors were similar before and after treatment. From the ROIs, signals in Exo $(+)$ tumors were significantly decreased $(0.56$-fold, $\mathrm{P}=0.0174)$, whereas signals in Exo (-) tumors did not decrease significantly. Tumor IHC showed that luciferase expression in Exo $(+)$ tumors was decreased compared to Exo (-) tumors. Moreover, Ephrin-A3 and PTP1B expression, which are miR-210 target proteins, was also decreased in Exo $(+)$ tumors compared to Exo (-) tumors (Figure 4e). However, VEGF and Ki67 levels were increased in Exo $(+)$ tumors compared to Exo (-) tumors. Based on western blot analysis of tumor tissues (Figure 4f), we confirmed that results were similar to IHC findings and showed that Ephrin-A3 and PTP1B protein levels were reduced in Exo $(+)$ tumors, while VEGF was increased in Exo $(+)$ tumors.

\section{DISCUSSION}

In this study, we developed a luciferase-based reporter vector to monitor miR-210. We demonstrated that exosomes from hypoxic tumor cells can transfer miRNA-210 to normoxic tumor/or endothelial cells and that exosomal miR-210 inhibited target genes and promoted angiogenesis in recipient cells.

Recently, exosomes have been actively investigated as novel messengers in cell-to-cell communication. The lipid bilayer of exosomes ensures the stability of their contents by protecting RNAs and proteins from degradation by circulating nucleases and proteases [1619]. Because of their unique physiological characteristics, 
Three copies of miR-210 target sequence

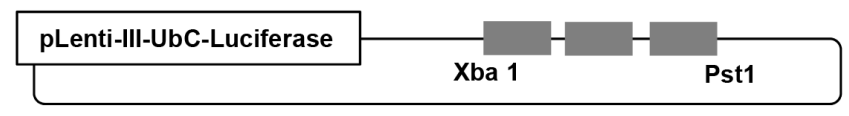

miR210:5' CUGUGCGUGUGACAGCGgCUGA

Forward: 5'- TCTAGAaatctagtTCAGCCGCTGTCACACGCACAGtagtaTCAGCCGCTGTCACA CGCACAGtagtaTCAGCCGCTGTCACACGCACAGCTGCAG-3'

Reverse: 5'- CTGCAGCTGTGCGTGTGACAGCGGCTGAtactaCTGTGCGTGTGACAGCGGCTGA tactaC TGTGCGTGTGACAGCGGCTGAactagattTCTAGA-3'

b

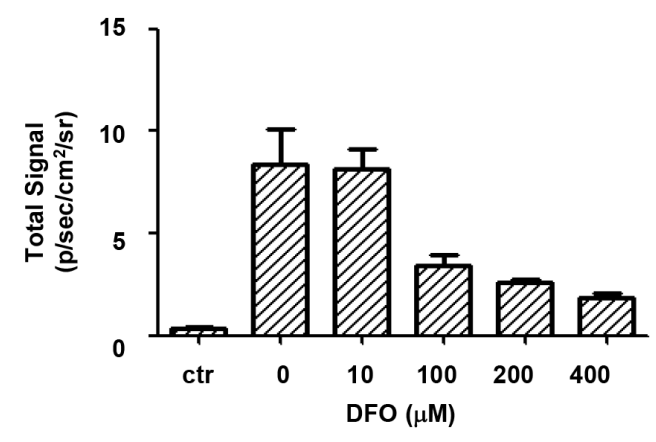

C

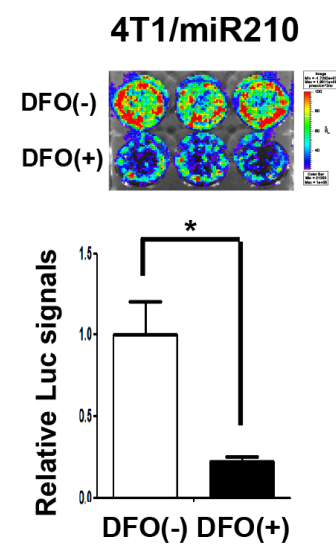

d

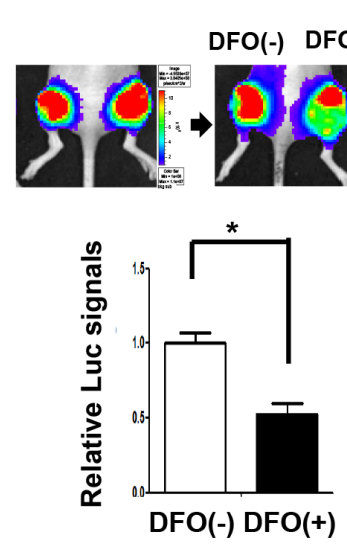

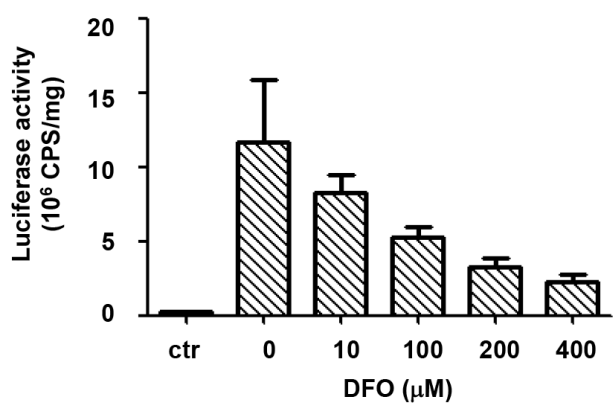

e
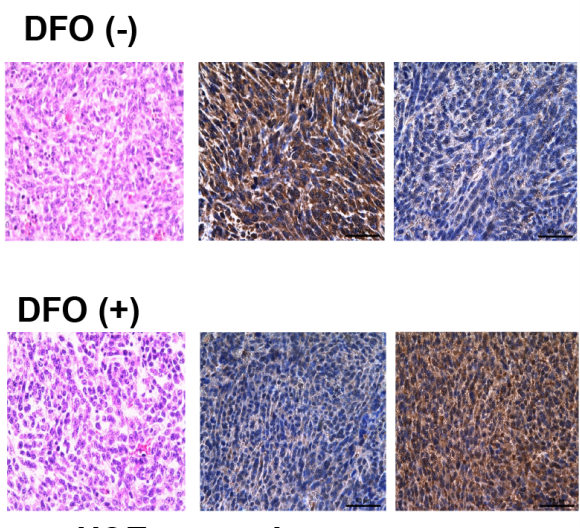

HIF-1 $\alpha$

Figure 3: Imaging miR-210 expression by DFO-induced hypoxia in 4T1 cells. a. Design of miR-210 reporter construct to image miR-210 expression. 4T1-luc2/miR-210 cells that express a miR-210 reporter vector were established, and luciferase signals from these cells could be turned off by the binding of miR-210. b. Reporter activity assay in 4T1 cells. IVIS images and luciferase assays showed that luciferase signals (left) and luciferase activity (right) were decreased by DFO treatment in a dose-dependent manner. c. Bioluminescence imaging showed that luciferase (Luc) signals from $400 \mu \mathrm{M}$ of DFO-treated cells were significantly decreased. d. Bioluminescence imaging of 4T1/miR-210 tumor-bearing mice; luciferase signals from the DFO $(+)$ tumor were significantly decreased $(n=3)$. DFO $(400 \mu M)$ was directly injected into the 4T1/miR-210 tumor. e. Immunohistochemistry of the DFO (+/-) 4T1/miR-210 tumor. Luciferase expression in DFO (+) tumors was decreased compared to DFO (-) tumors, whereas HIF- $1 \alpha$ expression in DFO (+) tumors was increased compared to DFO (-) tumors. $* \mathrm{P}<0.05$. 
a

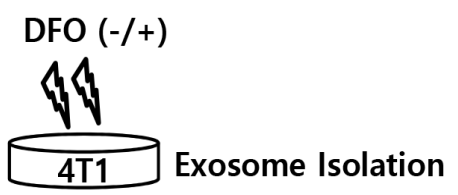

Exo (-) from DFO untreated cells Exo(+) from DFO treated cells

b
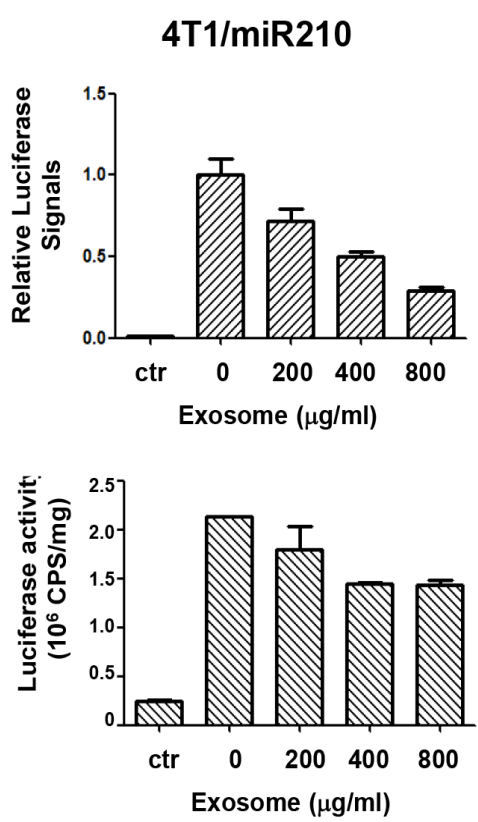

C
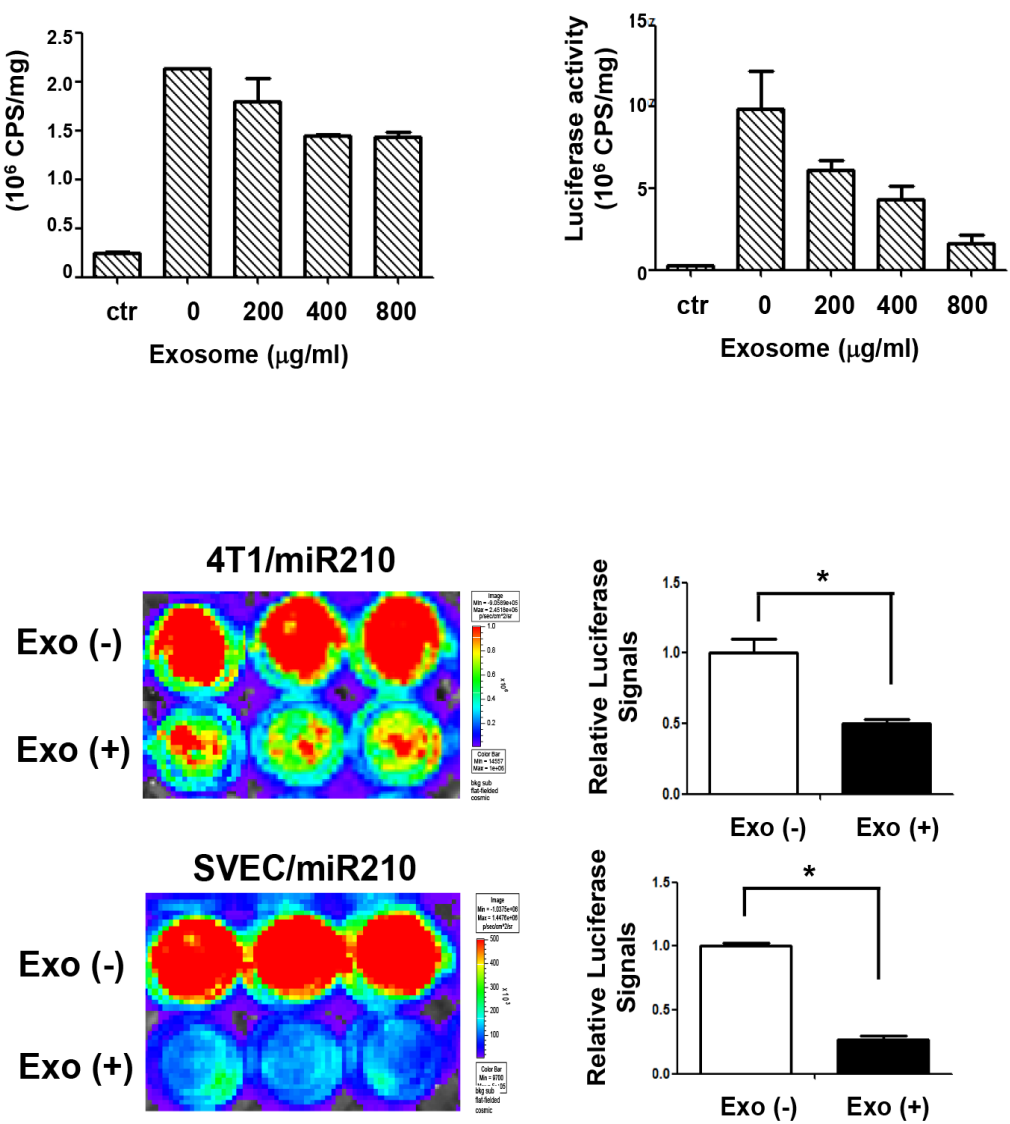

in vitro

in vivo

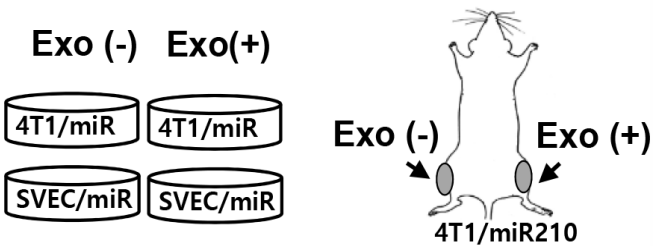

Figure 4: In vitro and in vivo miR-210 activation by hypoxic exosomes. a. Experimental scheme for imaging miR-210 activation by DFO-induced hypoxic exosomes. Exosomes from DFO-treated 4T1 cells were designated as EXO (+), and exosomes from PBS-treated 4T1 cells were designated as EXO (-). b. Both bioluminescence images and the luciferase assay showed a dose-dependent decrease in luciferase activity in cells treated with hypoxic exosomes (Exo (+)). c. Bioluminescence imaging of 4T1/miR210 and SVEC/miR210 cells after treatment with exosomes from DFO-treated/or -untreated 4T1 cells.

(Continued) 
d
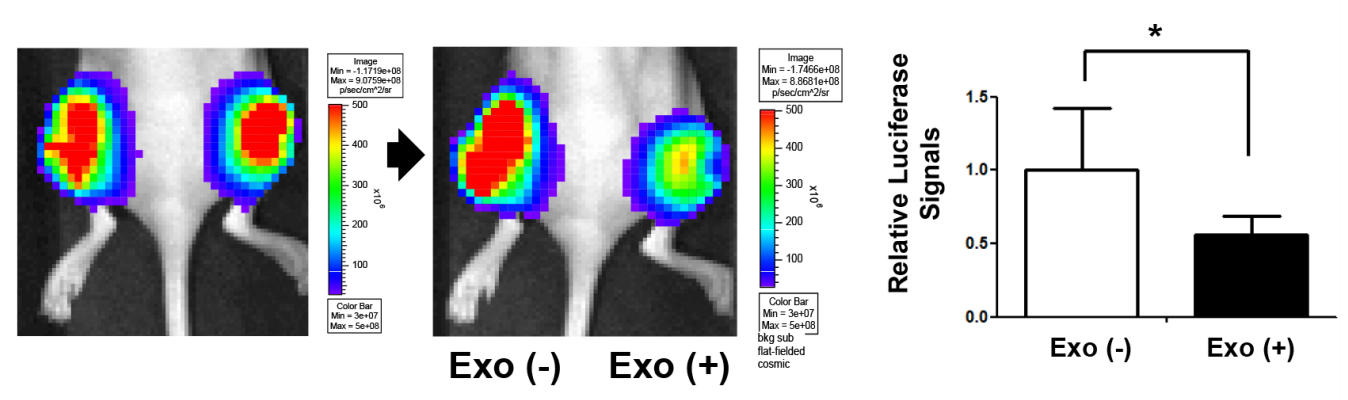

$\mathbf{e}$
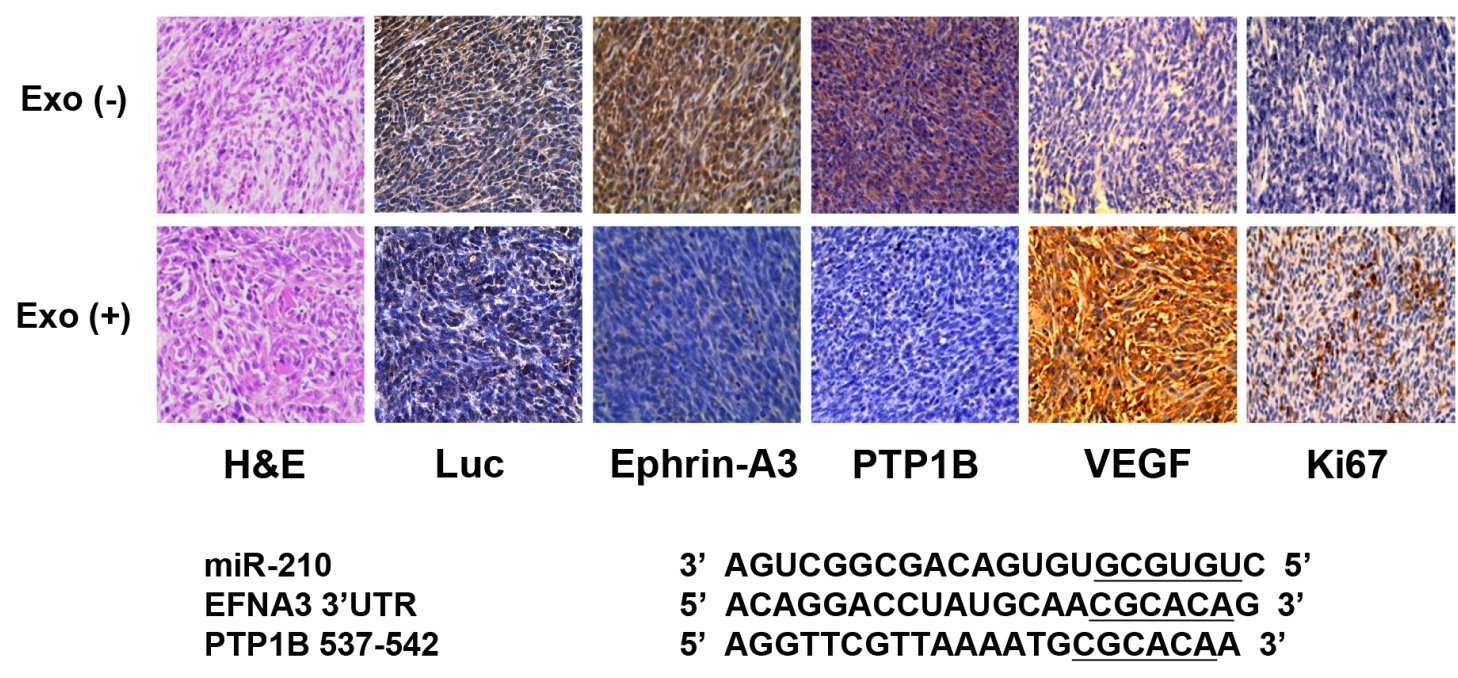

Ephrin-A3

PTP1B

VEGF

Ki67

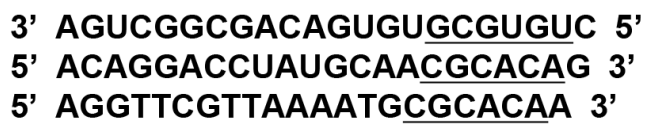

$\mathbf{f}$
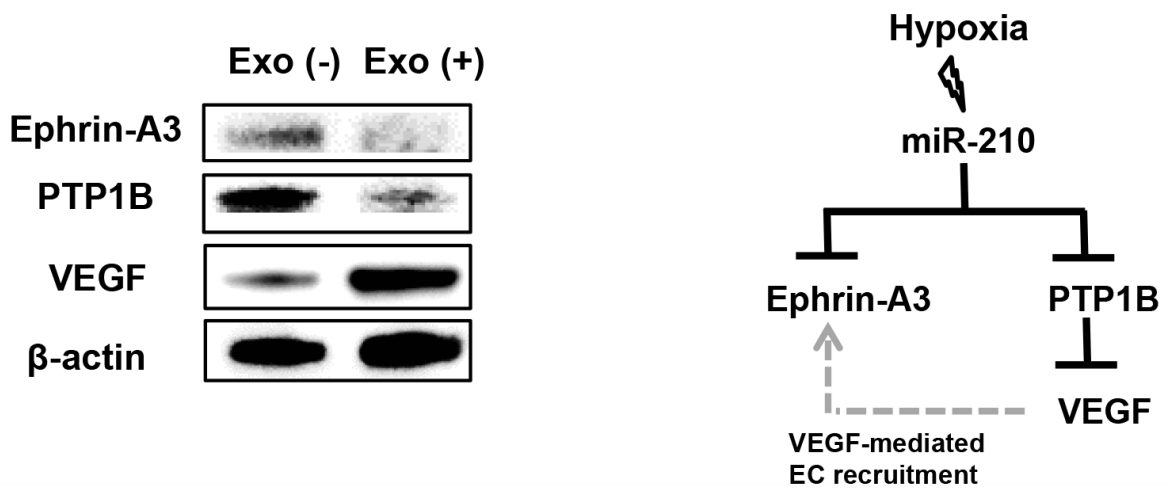

Figure 4 (Continued): d. Bioluminescence imaging of the 4T1/miR210 tumor after intra-tumoral treatment with exosomes $(400 \mu \mathrm{g})$ from DFO-treated or -untreated 4T1 cells $(n=4)$. e. Immunohistochemistry of 4T1/miR210 tumor after treatment with exosomes. Expression of luciferase and miR-210 targets, such as Ephrin-A3 and PTP1B, was decreased in Exo (+) tumors, while expression of VEGF and Ki67 was increased in Exo $(+)$ tumors. f. Western blotting of miR-210 target proteins and angiogenesis factor VEGF from 4T1/miR210 tumor tissues and their possible interactions. $* \mathrm{P}<0.05$. 
considerable attention has been given to the use of exosomes for diagnostic and therapeutic applications [2022]. Fluorescence images of exosomes in a tumor-grafted mouse show localization of exosomes in tumors (Figure 1b), suggesting tumor tropism of exosomes. Because exosomes are bioavailable, well-tolerable, targetable, and membrane-permeable, they are ideal candidates for delivery of miRNA, proteins, drugs, and other molecules to tumors.

Hypoxia is an important feature in tumors with malignant phenotypes and poor prognoses [23, 24]. Hypoxic tumors may communicate with surrounding tumor and non-tumor cells through exosomes to induce phenotypes that are more malignant. In particular, miR210 , which is induced by hypoxia, is associated with tumor progression, angiogenesis, and metastasis [25]. Our data show that exosomes from the serum of hypoxic tumorbearing mice had high miR-210 levels compared to normal mouse serum (Figure 1d). These results indicate that miR210 from circulating exosomes in the serum can be used as a potential biomarker for hypoxic tumors. In particular, our results suggest that exosome-mediated systemic transfer of miR-210 could influence nearby cells to produce a more favorable environment for tumor survival.

These results also show that exosomal miR210 from hypoxic cancer cells can be transferred to various types of recipient cells, such as epithelial cells, immune cells, and mesenchymal stem cells. It is possible that miRNA of exosomes from cancer cells can be transferred not only to surrounding cancer cells but also to neighboring stromal cells [29]. Functional efficiency of exosome-mediated miR-210 seems to be different in cell types. When we treated 4T1 and SVEC cells with the same amount of exosomes (Figure 4c), changes in luciferase signals between 4T1 and SVEC cells were quite different, suggesting that efficiency of exosome-mediated miR-210 functionality is dependent on cell type. The mode, effect, and clinical significance of exosome transfer to these microenvironment cells should be clarified.

Various target genes of miR-210, such as Ephrin-A3, PTP1B, HOXA1, and FGFRL1 [7], were reported, but we selected Ephrin-A3 and PTP1B because of their association with VEGF signaling and angiogenesis [26, 27]. Results show that Ephrin-A3 and PTP1B levels in tumors were decreased by treatment with exosomes containing miR-210. In contrast, VEGF levels in tumors were increased by treatment of exosomes with miR-210 (Figure 4e). Since Ephrin-A3 and PTP1B play important roles in the development of vascular remodeling [28], we speculated that downregulation of Ephrin-A3 and PTP1B through exosomal miR-210 could be correlated with angiogenic responses, resulting in formation of new capillaries and tubular structures.

Figure 5 shows that hypoxic tumor cells release miR-210 containing exosomes and that these exosomes are transferred to neighboring recipient cells. We found that transfer of exosomal miR-210 from hypoxic cells results in inhibition of miR-210 target genes, such as Ephrin-A3 and PTP1B, in neighboring cells in the tumor microenvironment. Vascular changes by down-regulation of Ephrin-A3 and PTP1B could increase VEGF and promote VEGF-mediated endothelial cell recruitment.

Although we only showed one example of exosomemediated transfer of hypoxia-induced genetic material

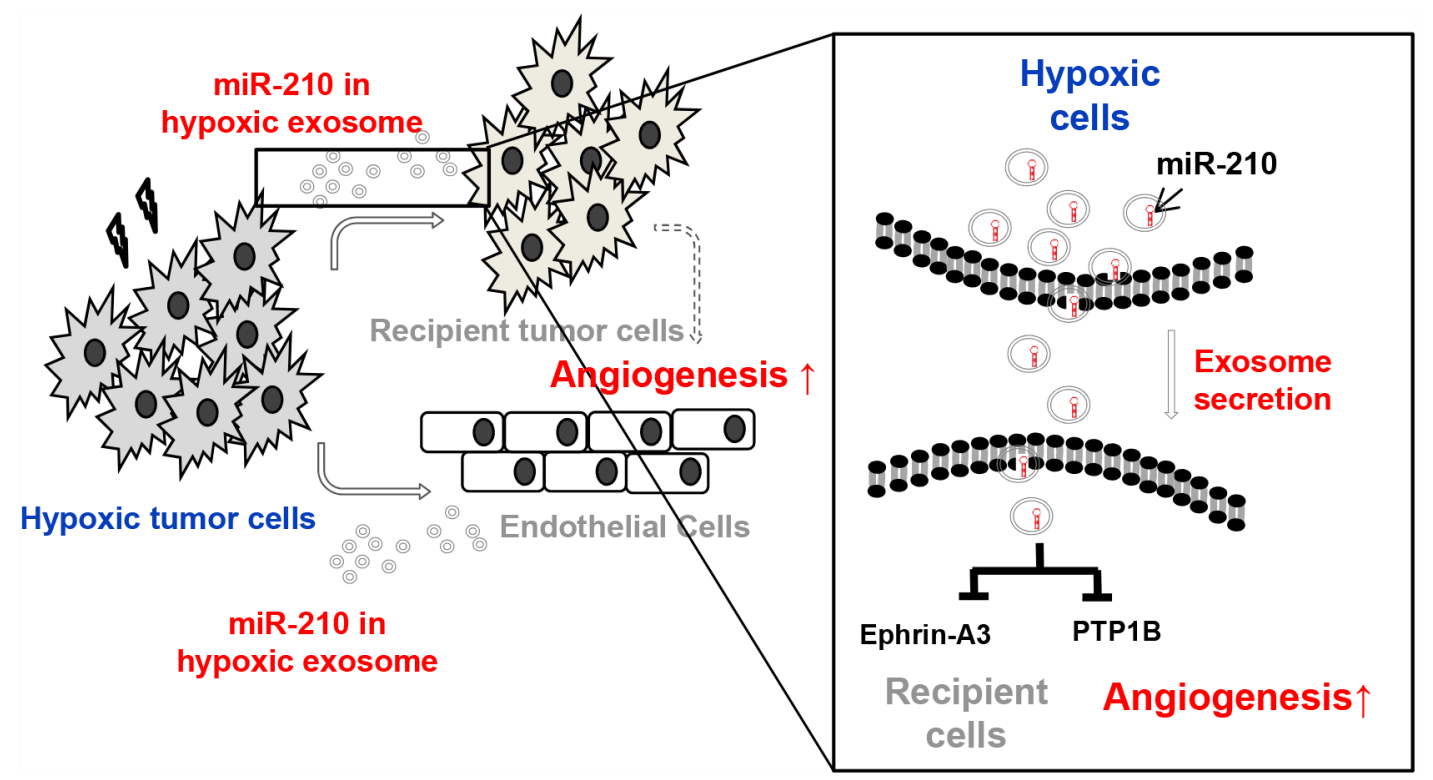

Figure 5: Summary. Hypoxic tumor cells release exosomes containing miR-210 and transfer them to neighboring cells in the tumor microenvironment. Recipient cells showed inhibition of miR-210 target genes, such as Ephrin-A3 and PTP1B, which influence changes in vascular structure to promote angiogenesis. 
(miR-210), it is important to visualize exosome-mediated transfer of other hypoxia-induced miRs, such as miR26, miR-31, miR107, and miR-424 [30-33] and their effects on the tumor microenvironment to investigate exosome-mediated malignant phenotype transfer of hypoxic cancer cells. Modifying imaging systems with other miRNA binding sites and monitoring cell-to-cell communication through exosomes will provide better knowledge to understand the role of exosomes in the tumor microenvironment.

\section{MATERIALS AND METHODS}

\section{Establishment of stable cell lines and hypoxic exposure}

A mouse breast cancer cell line (4T1) and endothelial cells (SVEC) were grown as monolayer cultures in RPMI-1640 medium supplemented with 1\% antibiotic-antimycotic mix and $10 \%$ fetal bovine serum (FBS). Cells were infected by retroviruses with reporter gene vectors $\mathrm{pCMV}-\mathrm{luc} 2 / \mathrm{miR}-210$ in which luciferase signals could be turned off by the binding of miR-210 to triplicates of the miR-210 binding site at the $3^{\prime}$ end of luc2 mRNA. Stable cell lines were selected by treatment with puromycin $(2 \mathrm{~g} / \mathrm{mL})$ for 2 weeks. $4 \mathrm{~T} 1$ cell lines with pCMV-luc2/miR-210 were labeled 4T1/miR210. Hypoxic conditions were induced with deferoxamine mesylate (DFO) (Sigma-Aldrich, St Louis, MO, USA) at $37^{\circ} \mathrm{C}$ in a $5 \% \mathrm{CO}_{2}$ humidified environment.

\section{Exosome purification}

4T1 cells $\left(1 \times 10^{6}\right.$ cells $)$ were cultured in conditioned medium. After $48 \mathrm{~h}$, exosomes were isolated from the culture medium using ultracentrifugation $(10,000$ $\times g$ ) or an exosome purification kit (ExoQuick ${ }^{\mathrm{TM}}$, System Bioscience, Mountain View, CA, USA). Exosome pellets were washed and resuspended with PBS. Proteins from exosome pellets and lysed cells were obtained by $1 \mathrm{X}$ RIPA buffer $(25 \mathrm{mM}$ Tris- $\mathrm{HCl} \mathrm{pH}$ 7.6, $150 \mathrm{mM} \mathrm{NaCl}, 1 \% \mathrm{NP}-$ $40,1 \%$ sodium deoxycholate, $0.1 \%$ SDS) with a cocktail of protease inhibitors (Roche, Nutley, NJ, USA). Protein concentrations were measured using a BCA protein assay kit (Pierce, Rockford, IL, USA).

\section{Transmission electron microscopy and NanoSight}

Exosomes were fixed with $2 \%$ glutaraldehyde (GAA) overnight at $4{ }^{\circ} \mathrm{C}$ and deposited by a copper grid (300 mesh covered with carbon). Size of exosomes was analyzed using transmission electron microscopy (TEM) images obtained using a JEOL (JEM 1400) transmission electron microscope at $80 \mathrm{keV}$ and measured with NanoSight (NanoSight Ltd., Malvern, UK).

\section{Western blotting}

Proteins $(20 \mu \mathrm{g})$ were separated using bis-Tris-HClbuffered $4 \%-12 \%$ gradient polyacrylamide gels (Invitrogen, Carlsbad, CA, USA) and blotted onto PVDF membranes (Millipore, Watford, UK). Membranes were blocked with $3 \%$ skim milk in TBS-T (20 mM Tris, $0.1 \%$ Tween 20, and $137 \mathrm{mM} \mathrm{NaCl}$ ) at room temperature for $1 \mathrm{~h}$. Primary antibodies were incubated overnight at $4{ }^{\circ} \mathrm{C}$ as follows: anti-AIP1/Alix (1:250 dilution; BD Biosciences, San Jose, CA, USA), anti-CD63 (1:500 dilution; System Bioscience, Mountain View, CA, USA), anti-CD9 (1:500 dilution; System Bioscience, Mountain View, CA, USA), and anticalnexin (1:500 dilution; Santa Cruz Biotechnology, Santa Cruz, CA, USA). Membranes were then incubated with secondary antibodies at room temperature for $1 \mathrm{~h}$ after washing three times with TBS-T. Secondary antibodies were used as follows: anti-mouse for AIP1/Alix (1:2,000 dilution; Invitrogen-Molecular Probes, Eugene, Oregon), anti-rabbit for CD63 and CD9 (1:2,000 dilution; System Bioscience, Mountain View, CA, USA) and anti-goat for calnexin (1:2,000 dilution; Invitrogen-Molecular Probes, Eugene, Oregon). Immuno-reactive bands were visualized using ECL reagents (Roche, Nutley, NJ, USA) and imaged using the LAS-3000 imaging system (Fuji Film, Tokyo, Japan).

\section{Fluorescence exosome imaging in vitro}

A stock solution of the lipophilic tracers (Invitrogen, Carlsbad, CA, USA) DiI (red fluorescence; $\mathrm{Ex}^{565 \mathrm{~nm}}, \mathrm{Em}^{594}$ $\mathrm{nm}$ ) and $\mathrm{DiO}$ (green fluorescence; $\mathrm{Ex}^{484 \mathrm{~nm}}, \mathrm{Em}^{501 \mathrm{~nm}}$ ) were prepared in ethanol and DMSO. Exosomes isolated from culture medium were incubated with DiO and DiI $(1 \mu \mathrm{M})$ dye for $30 \mathrm{~min}$ at $37^{\circ} \mathrm{C}$. Exosomes were then washed with PBS and purified using ExoQuick ${ }^{\mathrm{TM}}$. Fluorescently labeled exosomes $(20 \mu \mathrm{g} / \mathrm{mL})$ were used to treat tumor cells (4T1), endothelial cells (SVEC), macrophages (Raw264.7), stem cells (mBs-MSC), fibroblasts (3T3), and dentritic cells (JAWS2). Fluorescent exosomes in cells were detected using a Zeiss LSM510 META confocal imaging system (Carl Zeiss, Thornwood, CA, USA). We also constructed a pCMV driven GFP/RFP-tagged CD9 vector and imaged exosomes with confocal microscopy.

\section{Cell viability assay}

To evaluate the cytotoxicity of DFO and exosomes in 4T1 and SVEC cells, cell viability assays were performed after $48 \mathrm{~h}$ of treatment with DFO $(0,200,400,800 \mu \mathrm{M})$ and exosomes $(0,200,400,800 \mu \mathrm{g} / \mathrm{mL})$. After incubation with Cell Counting Kit-8 (CCK-8) solution for $2 \mathrm{~h}$, the mean optical density (OD) at $450 \mathrm{~nm}$ was measured.

\section{Fluorescence labeling of exosomes in vivo}

To label exosomes with $\mathrm{Cy} 7,50 \mu \mathrm{g}$ of exosomes (total volume $100 \mu \mathrm{L}$ ) were mixed with $0.5 \mu \mathrm{g}$ of $\mathrm{Cy}^{\mathrm{TM}} 7$ 
monoNHS ester ( $5 \mu \mathrm{M}$, Sigma-Aldrich, MO, USA) for $10 \mathrm{~min}$ at $37^{\circ} \mathrm{C}$. ExoQuick ${ }^{\mathrm{TM}}$ was used to purify $\mathrm{Cy} 7-$ labeled exosomes, followed by centrifugation at 3,000 $\times g$ for $15 \mathrm{~min}$. Cy7-labeled exosomes were imaged with the Maestro $^{\mathrm{TM}}$ in-vivo fluorescence imaging system (Cambridge Research Instrumentation, Woburn, MA, USA).

\section{Tumor grafts in nude mice}

All procedures involving in vivo mouse studies were approved by the Institutional Animal Care and Use Committee (IACUC) at Seoul National University and complied with the Guide for the Care and Use of Laboratory Animals. 4T1 cells $\left(1 \times 10^{6}\right.$ cells $)$ were subcutaneously transplanted in the thighs of 6-week-old male BALB/c nu/nu mice weighing $20 \mathrm{~g}$ on average, and tumors were grown to $10 \mathrm{~mm}$ in diameter.

\section{Fluorescence imaging in vivo}

Cy7-labeled exosomes $(200 \mu \mathrm{g})$ were intravenously injected in tumor xenograft mice. Exosome signals were imaged using the IVIS200 imaging system (Xenogen Corp., Alameda, CA, USA) and a CCD camera.

\section{Quantification of miR-210 by RT-qPCR}

4T1 cells $\left(1 \times 10^{6}\right.$ cells $)$ were cultured in conditioned medium and treated with $400 \mu \mathrm{M}$ DFO for $48 \mathrm{~h}$. Blood from tumor-bearing mice was collected by cardiac puncture at $48 \mathrm{~h}$ after $400 \mu \mathrm{M}$ DFO treatment, and exosomes were harvested from the serum. Total RNA was extracted using TRIzol reagent (Invitrogen, Carlsbad, CA, USA). Quantification of miR-210 was performed with SYBR Green real-time PCR Master Mix and Mir-X ${ }^{\text {TM }}$ miRNA First-Strand Synthesis (TaKaRa, Otsu, Japan) according to the manufacturer's instruction. U6 was used as a housekeeping gene to standardize the initial miRNAs from a sample. Data are presented as fold downregulation or upregulation. Fold value $=2^{-\Delta \Delta \mathrm{Ct}}$, where $\Delta \Delta \mathrm{Ct}=(\mathrm{Ct}$ of gene of interest, treated-Ct of housekeeping gene, treated)-(Ct of gene of interest, control-Ct of housekeeping gene, control), and $\mathrm{Ct}$ was the number of threshold cycles. Primer sequences were used as follows: miR-210 forward 5'-CTGTGCGTGTGACAGCGGCTGA-3', HIF- $1 \alpha$ forward 5'-GCACAGGCCACATTCACG-3', and U6 forward 5'-TGGCCCCTGCGCAAGGATG-3'.

\section{Effects of hypoxic exosomes in SVEC cells}

To confirm the effects of hypoxic exosomes, SVEC cells were treated with hypoxic exosomes from 4T1 cells $(400 \mu \mathrm{g} / \mathrm{mL})$ for $48 \mathrm{~h}$. Wound healing assay was performed and capillary-like structures were confirmed by microscopy.

\section{Imaging of miR-210 in vitro}

4T1-luc2/miR210 cells $\left(1 \times 10^{5}\right.$ cells $)$ were seeded in 24-well plates. Cells were treated with DFO $(400 \mu \mathrm{M})$ or exosomes $(400 \mu \mathrm{g})$ isolated from cells treated with DFO $(400 \mu \mathrm{M})$. After $48 \mathrm{~h}$, cells were treated with $100 \mu \mathrm{L}$ of luciferin $(0.3 \mu \mathrm{g} / \mu \mathrm{L})$ before bioluminescence imaging. Images were obtained using the IVIS200 imaging system equipped with a CCD camera (Xenogen Corp., Alameda, CA, USA). Bioluminescent images were analyzed using LIVINGIMAGE V. 2.50.1 software (Xenogen Corp., Alameda, CA, USA).

\section{Luciferase assays in vitro}

Luciferase enzyme assays were performed using luciferase assay kits (Applied Biosystems, Carlsbad, CA, USA). Cells were plated and treated with DFO or exosomes, as previously described. After $48 \mathrm{~h}$, the wells were washed twice with PBS and lysis solution was added to each well. Cell lysates were then transferred to a microplate. Bioluminescence was measured using a Wallac 1420 VICTOR3 V plate reader (PerkinElmer Life and Analytical Sciences, Shelton, CT, USA).

\section{Imaging of miR-210 in vivo}

Mice were injected intratumorally with PBS (in the left thigh) and $400 \mu \mathrm{M}$ of DFO (in the right thigh). In the exosome treatment group, mice were injected intratumorally with exosomes $(400 \mu \mathrm{g} / \mathrm{mL})$ in the right thigh. After $48 \mathrm{~h}$, mice were intraperitoneally injected with $100 \mu \mathrm{L}$ of the luciferase substrate luciferin $(30 \mu \mathrm{g} / \mu \mathrm{L})$. Mice were anesthetized with isoflurane and images were obtained using the IVIS200 imaging system, as described.

\section{Immunohistochemistry (IHC)}

Tumor tissues were fixed in $3.7 \%$ paraformaldehyde for $24 \mathrm{~h}$ and embedded with paraffin, prepared as $4-\mu \mathrm{m}$ sections, and mounted on slides. Antigens were retrieved by boiling in citrate buffer (DakoCytomation, Glostrup, Denmark) for $5 \mathrm{~min}$. Primary antibodies were incubated overnight at $4{ }^{\circ} \mathrm{C}$ as follows: anti-luciferase (1:500 dilution; Abcam, Cambridge, UK), anti-HIF-1 $\alpha$ (1:100 dilution; Novus Biologicals, Littleton, CO, USA), and anti-Ephrin-A3 (1:500 dilution; Santa Cruz Biotechnology, Santa Cruz, CA, USA). Secondary antibodies were used as follows: biotinylated anti-goat for luciferase (1:500 dilution; Dako, Glostrup, Denmark) and biotinylated anti-mouse for HIF-1 $\alpha$ and Ephrin-A3 (1:500 dilution; Dako, Glostrup, Denmark). An avidin-biotin peroxidase complex was used to amplify the signal, followed by development using DAB and counterstaining with hematoxylin. 


\section{Deparaffinization and protein extraction}

Deparaffinization and protein extraction were performed using Qiagen's Qproteome FFPE Tissue Kit (Qiagen, Hilden, Germany). All FFPE samples were fixed in $3.7 \%$ paraformaldehyde for $24 \mathrm{~h}$ and used for protein extraction. Six slides each with $4 \mu \mathrm{m}$ sections and areas of up to $100 \mathrm{~mm}^{2}$ were randomly selected. Briefly, paraffin tissue slides were removed by immersion in xylene for $10 \mathrm{~min}$ at room temperature. Tissues were rehydrated with a graded ethanol series $(100 \%, 95 \%$, and $70 \%)$ followed by double-distilled water. Using a needle, areas of tissue were excised and transferred to $1.5-\mathrm{mL}$ collection tubes. Extraction buffer and $\beta$-mercaptoethanol (Sigma-Aldrich, St Louis, MO, USA) were added to the tube at the recommended volume and incubated at $100^{\circ} \mathrm{C}$ for $20 \mathrm{~min}$ in order to reduce disulfide bonds, followed by incubation at $80^{\circ} \mathrm{C}$ for $2 \mathrm{~h}$ to ensure maximal protein extraction. Finally, the product was centrifuged for $15 \mathrm{~min}$ at $14000 \times \mathrm{g}$, and protein extracts were stored at $-20^{\circ} \mathrm{C}$. Protein concentration was measured using the $\mathrm{BCA}$ protein assay kit (Pierce, Rockford, IL, USA) according to the manufacturer's protocol.

\section{Western blot from FFPE tissue}

Proteins $(50 \mu \mathrm{g})$ were extracted from FFPE tissues and separated as mentioned. Primary antibodies were incubated overnight at $4^{\circ} \mathrm{C}$ as follows: anti-Ephrin-A3 (1:50 dilution; Santa Cruz Biotechnology, Santa Cruz, CA, USA), anti-PTP1B (1:200 dilution; Abcam, Cambridge, UK), and anti-VEGF (1:200 dilution; Abcam, Cambridge, UK). Secondary antibodies were used as follows: antirabbit for Ephrin-A3, PTP1B, and VEGF (1:2,000 dilution, Cell Signaling Technology, Danvers, MA, USA). Beta-actin was used as a loading control.

\section{Statistical analysis}

All results are presented as the mean $\pm \mathrm{SD}$. Student's unpaired t-test was used to determine statistical significance, and $\mathrm{P}$-values $<0.05$ were considered statistically significant.

\section{ACKNOWLEDGMENTS}

This work was supported by the National Research Foundation (NRF) for the Global Core Research Center (GCRC) funded by the Ministry of Science and ICT \& Future Planning (MSIP) (No. 2011-0030001). It was also supported by a grant (HI13C0826, HI14C1072, HI15C2971) from the Korea Health Technology R\&D Project, Ministry of Health \& Welfare, Republic of Korea, SNUH Research fund 03-2013-0420 and the KRIBB Research Initiative Program.

\section{CONFLICTS OF INTEREST}

The authors have declared that they have no competing interest.

\section{REFERENCES}

1. Hanahan D, Weinberg RA. Hallmarks of Cancer: The Next Generation. Cell. 2011; 144:646-674.

2. Ruan K, Song G, Ouyang G. Role of hypoxia in the hallmarks of human cancer. J Cell Biochem 2009; 107:1053-1062.

3. Mees G, Dierckx R, Vangestel C, Van de Wiele C. Molecular imaging of hypoxia with radiolabelled agents. Eur J Nucl Med Mol Imaging 2009; 36:1674-1686.

4. Krohn KA, Link JM, Mason RP. Molecular imaging of hypoxia. J Nucl Med 2008; 49:129S-148S.

5. Wittmann J, Jack HM. Serum microRNAs as powerful cancer biomarkers. Biochim Biophys Acta 2010; 1806:200-207.

6. Iorio MV, Croce CM. MicroRNA dysregulation in cancer: diagnostics, monitoring and therapeutics. EMBO Mol Med 2012; 4:143-159.

7. Mathew LK, Simon MC. mir-210: a sensor for hypoxic stress during tumorigenesis. Mol Cell 2009; 35:737-738.

8. Huang X, Le QT, Giaccia AJ. MiR-210--micromanager of the hypoxia pathway. Trends Mol Med 2010; 16:230-237.

9. Thery C, Zitvogel L, Amigorena S. Exosomes: composition, biogenesis and function. Nat Rev Immunol 2002; 2:569-579.

10. Raposo G, Stoorvogel W. Extracellular vesicles: exosomes, microvesicles, and friends. Cell Biol. 2013; 200:373-383.

11. Quail DF, Joyce JA. Microenvironmental regulation of tumor progression and metastasis. Nat. Med. 2013; 19:1423-1437.

12. Record M, Subra C, Silvente-Poirot S, Poirot M. Exosomes as intercellular signalosomes and pharmacological effectors. Biochem. Pharmacol. 2011; 81:1171-1182.

13. Hannafon BN, Ding WQ. Intercellular Communication by Exosome-Derived microRNAs in Cancer. Int. J. Mol. Sci. $2013 ; 14: 14240-14269$.

14. King HW, Michael MZ, Gleadle JM. Hypoxic enhancement of exosome release by breast cancer cells. BMC Cancer 2012; $12: 421$.

15. Ho AS, Huang X, Cao H, Christman-Skieller C, Bennewith K, Le QT, Koong AC. Circulating miR-210 as a Novel Hypoxia Marker in Pancreatic Cancer. Transl Oncol 2010; 3:109-113.

16. Chou J, Shahi P, Werb Z. microRNA-mediated regulation of the tumor microenvironment. Cell Cycle 2013; 12:3262-3271.

17. Ochiya T, Lotvall J. Exosome as a novel shuttle for innovation. Preface. Adv Drug Deliv Rev 2013; 65:v. 
18. Umezu T, Ohyashiki K, Kuroda M, Ohyashiki JH. Leukemia cell to endothelial cell communication via exosomal miRNAs. Oncogene 2013; 32:2747-2755.

19. Hannafon BN, Ding WQ. Intercellular Communication by Exosome-Derived microRNAs in Cancer. Int $\mathrm{J}$ Mol Sci $2013 ; 14: 14240-14269$.

20. Tickner JA, Urquhart AJ, Stephenson SA, Richard DJ, O'Byrne KJ. Functions and therapeutic roles of exosomes in cancer. Front. Oncol 2014; 4:127.

21. Tan A, Rajadas J, Seifalian AM. Exosomes as nanotheranostic delivery platforms for gene therapy. Adv Drug Deliver Rev 2013; 65:357-367.

22. Lasser C. Exosomal RNA as biomarkers and the therapeutic potential of exosome vectors. Expert Opin Biol Ther 2012; 12:S189-197.

23. Arvelo F, Cotte C. Hypoxia in cancer malignity. Invest Clin, 2009; 50:529-546.

24. Vaupel P, Mayer A. Hypoxia in cancer: significance and impact on clinical outcome. Cancer Metastasis Rev 2007; $26: 225-239$

25. Dang K, Myers KA. The role of hypoxia-induced miR-210 in cancer progression. Int J Mol Sci 2015; 16:6353-6372.

26. Fasanaro P, D'Alessandra Y, Di Stefano V, Melchionna R, Romani S, Pompilio G, Capogrossi MC, Martelli F. MicroRNA-210 modulates endothelial cell response to hypoxia and inhibits the receptor tyrosine kinase ligand Ephrin-A3. J Biol Chem 2008; 283:15878-15883.
27. Gee HE, Ivan C, Calin GA, Ivan M. HypoxamiRs and cancer: from biology to targeted therapy. Antioxid Redox Sign 2014; 21:1220-1238.

28. Kuijper S, Turner CJ, Adams RH. Regulation of angiogenesis by Eph-ephrin interactions. Trends Cardiovasc Med 2007; 17:145-151.

29. Catalano V, Turdo A, Di Franco S, Dieli F, Todaro M, Stassi G. Tumor and its microenvironment: A synergistic interplay. Semin Cancer Biol. 2013.

30. Wilson WR, Hay MP. Targeting hypoxia in cancer therapy. Nat Rev Cancer 2011; 11: 393-410.

31. Ghosh G, Subramanian IV, Adhikari N, Zhang X, Joshi HP, Basi D, Chandrashekhar YS, Hall JL, Roy S, Zeng Y, Ramakrishnan S. Hypoxia-induced microRNA-424 expression in human endothelial cells regulates HIF-alpha isoforms and promotes angiogenesis. J Clin Invest 2010; 120:4141-4154.

32. Liu CJ, Tsai MM, Hung PS, Kao SY, Liu TY, Wu KJ, Chiou SH, Lin SC, Chang KW. miR-31 ablates expression of the HIF regulatory factor FIH to activate the HIF pathway in head and neck carcinoma. Cancer Res 2010; 70:1635-1644.

33. Kulshreshtha R, Ferracin M, Wojcik SE, Garzon R, Alder H, Agosto-Perez FJ, Davuluri R, Liu CG, Croce CM, Negrini M, Calin GA, Ivan M. A microRNA signature of hypoxia. Mol Cell Biol. 2007; 27:1859-67. 